\title{
Racemase activity effected by two dehydrogenases in sulfolactate degradation by Chromohalobacter salexigens: purification of $(S)$-sulfolactate dehydrogenase
}

\author{
Karin Denger and Alasdair M. Cook \\ Department of Biology, The University, D-78457 Konstanz, Germany
}

\begin{abstract}
Chromohalobacter salexigens DSM 3043, whose genome has been sequenced, is known to degrade $(R, S)$-sulfolactate as a sole carbon and energy source for growth. Utilization of the compound(s) was shown to be quantitative, and an eight-gene cluster (Csal_1764-Csal_1771) was hypothesized to encode the enzymes in the degradative pathway. It comprised a transcriptional regulator (SuyR), a Tripartite Tricarboxylate Transporter-family uptake system for sulfolactate (SlcHFG), two sulfolactate dehydrogenases of opposite sulfonate stereochemistry, namely novel SlcC and ComC [(R)-sulfolactate dehydrogenase] [EC 1.1.1.272] and desulfonative sulfolactate sulfo-lyase (SuyAB) [EC 4.4.1.24]. Inducible reduction of 3-sulfopyruvate, inducible Suy $A B$ activity and induction of an unknown protein were detected. Separation of the soluble proteins from induced cells on an anion-exchange column yielded four relevant fractions. Two different fractions reduced sulfopyruvate with $\mathrm{NAD}(\mathrm{P}) \mathrm{H}$, a third yielded SuyAB activity, and the fourth contained the unknown protein. The latter was identified by peptide-mass fingerprinting as $\mathrm{SlcH}$, the candidate periplasmic binding protein of the transport system. Separated SuyB was also identified by peptide-mass fingerprinting. ComC was partially purified and identified by peptide-mass fingerprinting. The $(R)$-sulfolactate that ComC produced from sulfopyruvate was a substrate for SuyAB, which showed that SuyAB is $(R)$-sulfolactate sulfo-lyase. SlcC was purified to homogeneity. This enzyme also formed sulfolactate from sulfopyruvate, but the latter enantiomer was not a substrate for SuyAB. SIcC was obviously (S)-sulfolactate dehydrogenase.
\end{abstract}

\section{INTRODUCTION}

3-Sulfolactate (hereafter sulfolactate) is a natural product in algae, animals, archaea, bacteria and plants (e.g. Bonsen et al., 1969; Danko et al., 2006; Graham \& White, 2002; Lee \& Benson, 1972; Roy et al., 2003; Shibuya et al., 1963; Weinstein \& Griffith, 1988). The only major textbook to refer to sulfolactate (Metzler, 2003) implies a role in the biosynthesis of sulfoquinovose (SQ; 6-deoxy-6-sulfo-Dglucopyranose, the polar headgroup of the plant sulfolipid sulfoquinovosyl diacylglycerol), whereas, following Benning's work on SQ biosynthesis (Benning, 2007), sulfolactate is known to be an intermediate in the manipulation of SQ to a range of plant (and algal) products such as alkaloids (Folkers et al., 1944), as suspected by Benson (Benson \& Lee, 1972). The widespread occurrence of sulfolactate has apparently led to considerable diversity in the degradation of the compound,

Abbreviations: ComC, $(R)$-sulfolactate dehydrogenase; $\mathrm{SlcC},(S)$-sulfolactate dehydrogenase; SICHFG, tripartite tricarboxylate transport (TTT)family transporter for sulfolactate; $S Q$, sulfoquinovose; SuyAB, sulfolactate sulfo-lyase. and three desulfonative pathways are already known. Whereas sulfolactate can be desulfonated by sulfolactate sulfo-lyase (SuyAB) [EC 4.4.1.24] in Paracoccus pantotrophus NKNCYSA (Rein et al., 2005), Roseovarius nubinhibens ISM has a bifurcated pathway (Denger et al., 2009) which involves both sulfoacetaldehyde acetyltransferase [EC 2.3.3.15] (Ruff et al., 2003) and (S)-cysteate sulfo-lyase (CuyA) [EC 4.4.1.25] (Denger et al., 2006). The pathway to $(S)$-cysteate in $R$. nubinhibens is incomplete, because a sulfolactate racemase (or its equivalent) has not been identified.

The work with $R$. nubinhibens yielded the first candidate for a sulfolactate transporter, SlcHFG; the second SlcHFG (Csal_1767 - Csal_1769) was found in Chromohalobacter salexigens DSM 3043 associated with SuyAB (Denger et al., 2009). The rarity of this transporter caused us to examine the relevant eight-gene cluster in C. salexigens (Csal_1764Csal_1771) (Fig. 1). The genes encoding the presumed transporter are sandwiched between suyAB (Csal_1765Csal_1766) and a pair of overlapping genes annotated to encode NAD(P)-coupled dehydrogenases (Csal_1770Csal_1771). The latter is an orthologue of ComC, $(R)$ - 
(S)-sulfolactate ${ }_{-\mathrm{O}_{3} \mathrm{~S}} \overbrace{\mathrm{OH}}^{\mathrm{O} H}$

(R)-sulfolactate

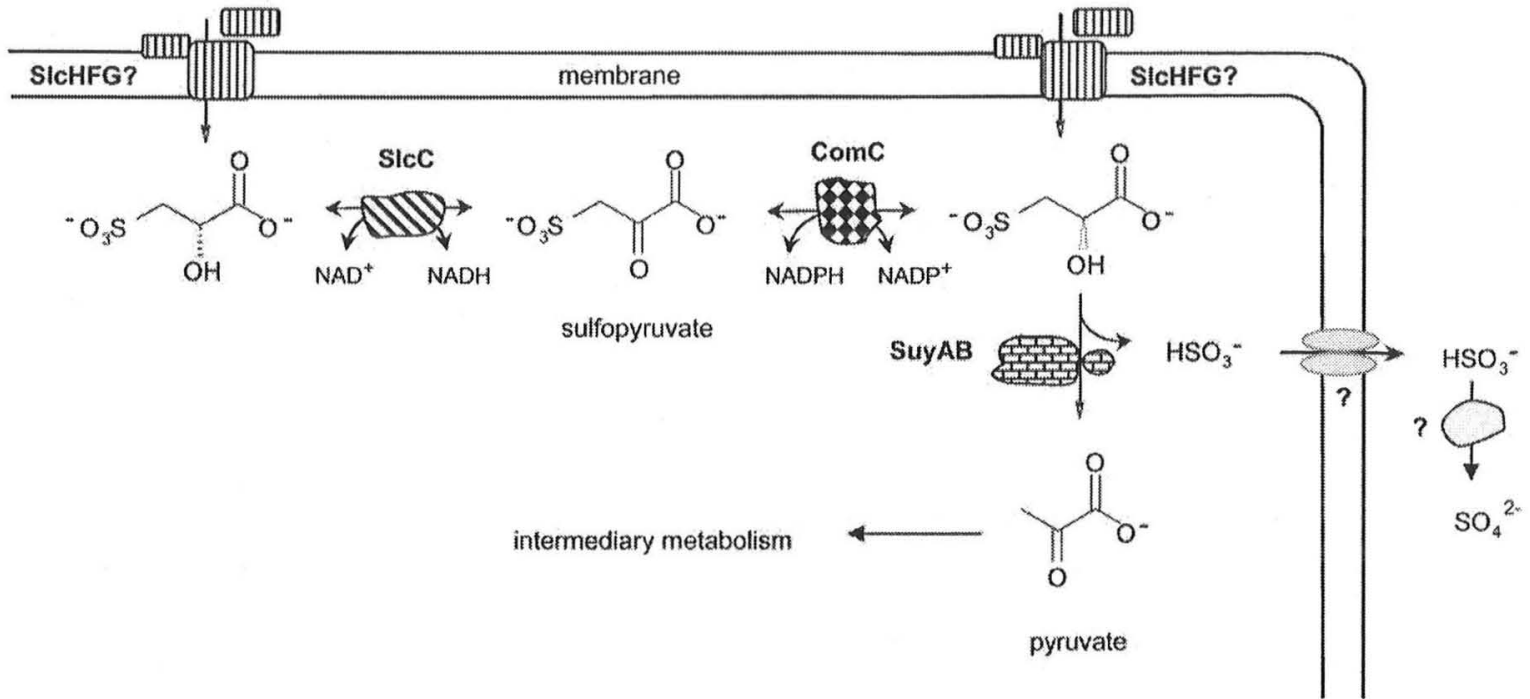

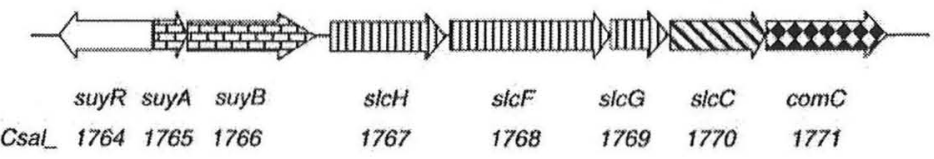

Fig. 1. Derived pathway of degradation of $(R, S)$-sulfolactate in C. salexigens DSM 3043 and the gene cluster encoding the pathway enzymes. The natures of the putative sulfite exporter and of the sulfite dehydrogenase are unknown.

sulfolactate dehydrogenase [EC 1.1.1.272] (Graupner et al., 2000; Irimia et al., 2004), so, requiring a racemaseequivalent, we hypothesized that Csal_1770 represents (S)-sulfolactate dehydrogenase (SlcC). The current annotation of Csal_1770 is SerA [(S)-3-phosphoglycerate dehydrogenase, EC 1.1.1.95] in serine biosynthesis. The eighth gene in the cluster is an orthologue of suyR, the putative transcriptional regulator of sulfolactate degradation in $P$. pantotrophus (Rein et al., 2005). This information allowed a simple degradative pathway for $(R, S)$-sulfolactate to be drafted (Fig. 1), and the unknown stereospecificity of SuyAB (Rein et al., 2005) to be deduced.

We now report supportive data for this pathway in $C$. salexigens DSM 3043. The paper concerns largely the initial scalar reaction in the pathway, that of novel $(S)$-sulfolactate dehydrogenase, which was purified and characterized.

\section{METHODS}

Chemicals. Racemic sulfolactate (Roy et al., 2003) and 3-sulfopyruvate (hereafter sulfopyruvate) (Denger et al., 2001) were synthesized as described in the papers cited. Initial work used the high quality sulfopyruvate (Denger et al., 2001), but the new preparations were of low purity, which did not allow reliable quantification of e.g. $K_{\mathrm{m}}$ values at the end of the project. Commercial chemicals were of the highest purity available and purchased from Sigma-Aldrich, Roth, Merck, Biomol or Fluka.

Organisms, growth medium and growth conditions. C. salexigens DSM 3043, a marine gammaproteobacterium (Arahal et al., 2001), was obtained from the German Culture Collection (DSMZ, Braunschweig, Germany). Cells were grown aerobically at $30{ }^{\circ} \mathrm{C}$ in Tris-buffered artificial seawater (Krejčík et al., 2008) supplemented with $10 \mathrm{mM}$ ammonium chloride and a seven-vitamin solution (Pfennig, 1978); the sole added source of carbon and energy was $10 \mathrm{mM}(R, S)$-sulfolactate or $20 \mathrm{mM}$ acetate. Precultures $(3 \mathrm{ml})$ were grown in $30 \mathrm{ml} \mathrm{screw}$-cap tubes in a roller. Growth experiments were done on the $30 \mathrm{ml}$ scale in $300 \mathrm{ml}$ Erlenmeyer flasks shaken at $30{ }^{\circ} \mathrm{C}$. Samples were taken at intervals to measure attenuance $(580 \mathrm{~nm})$, to assay protein, and to determine the concentrations of sulfolactate, sulfate or sulfite. Similar cultures were used to generate small amounts of cells for enzyme assays. Cultures (1 l) for protein purification were grown in 51 Erlenmeyer flasks on a shaker. Cells were harvested at $\mathrm{OD}_{580} 0.45$ (about $110 \mathrm{mg}$ protein $\mathrm{l}^{-1}$ ) by centrifugation $\left(15000 \mathrm{~g}, 20 \mathrm{~min}, 4^{\circ} \mathrm{C}\right)$, washed with $50 \mathrm{mM}$ Tris buffer, $\mathrm{pH}$ 9.0, and stored frozen. The same buffer served as extraction buffer. Cell-free extracts free of nucleic acids after DNase 
treatment $\left(0.05 \mathrm{mg}\right.$ DNase $\left.\mathrm{I} \mathrm{ml}^{-1}\right)$ were generated after disruption by three passages through a French pressure cell set at $140 \mathrm{MPa}$ (Junker et al., 1994). The membrane/particulate fraction was sedimented by ultracentrifugation $\left(220000 \mathrm{~g}, 30 \mathrm{~min}, 4{ }^{\circ} \mathrm{C}\right)$ and the supernatant fluid was called the soluble fraction.

Cupriavidus necator JMP134 (DSM 4058), Methylobacterium radiotolerans (DSM 1819) and Oligotropha carboxidovorans (DSM 1227) were purchased from the DSMZ, and Rhodococcus jostii RHAl was kindly supplied by W. W. Mohn (University of British Columbia, Vancouver, BC, Canada). Strains JMP134 and RHAl were grown in our standard freshwater medium (Thurnheer et al., 1986). Other organisms were grown in the salts medium recommended by DSMZ.

Enzyme assays. (S)-Sulfolactate dehydrogenase (SlcC) was routinely assayed photometrically $(365 \mathrm{~nm}$ ) at room temperature (about $23{ }^{\circ} \mathrm{C}$ ) as the sulfopyruvate-dependent oxidation of NADH. The reaction mixture contained, in $25 \mathrm{mM}$ Tris buffer, $\mathrm{pH} 9.0$, sulfopyruvate $(2 \mathrm{mM}), \mathrm{NADH}(0.25 \mathrm{mM})$ and protein $\left(2-200 \mu \mathrm{g} \mathrm{ml}^{-1}\right)$, with which the reaction was started. Variants of the assay were used to determine kinetic constants. ( $R$ )-sulfolactate oxidoreductase (ComC) [EC 1.1.1.272] was routinely assayed photometrically $(365 \mathrm{~nm})$ at room temperature as the sulfolactate-dependent reduction of $\mathrm{NADP}^{+}$. The reaction mixture contained, in $0.5 \mathrm{M}$ glycine $/ 0.4 \mathrm{M}$ hydrazine buffer, $\mathrm{pH} 9.0$, sulfolactate $(10 \mathrm{mM}), \mathrm{NADP}^{+}(2 \mathrm{mM})$ and protein $\left(2-200 \mu \mathrm{g} \mathrm{ml}^{-1}\right)$, with which the reaction was started. 3Phosphoglycerate dehydrogenase (SerA) [EC 1.1.1.95] was assayed under the same conditions, with 3-phosphoglycerate in place of sulfolactate. The reaction of each sulfolactate dehydrogenase could also be followed discontinuously in the presence of NADH or NADPH by ion chromatography by measuring the decrease (increase) of the sulfopyruvate (sulfolactate) concentration. In this case the concentration of sulfopyruvate was initially $0.5 \mathrm{mM}$. Sulfolactate sulfo-lyase (SuyAB) [EC 4.4.1.24] was assayed as release of sulfite from sulfolactate, with SuyAB from $P$. pantotrophus NKNCYSA as a positive control (Rein et al., 2005). The MOPS buffer was replaced with $50 \mathrm{mM}$ Tris buffer, $\mathrm{pH}$ 9.0. Sulfite dehydrogenase (SorAB) [EC 1.8.2.1] was assayed photometrically with $\mathrm{K}_{3} \mathrm{Fe}(\mathrm{CN})_{6}$ as electron acceptor (Reichenbecher et al., 1999) as modified elsewhere (Denger et al., 2008), and enzyme from Cupriavidus necator H16 served as positive control (Denger et al., 2008).

Separation and purification of enzymes. Soluble fraction of $C$. salexigens in $25 \mathrm{mM}$ Tris/sulfate buffer, $\mathrm{pH} 9.0$ was loaded onto an anion-exchange chromatography column (Mono Q, HR 10/10, Pharmacia) at a flow rate of $1.0 \mathrm{ml} \mathrm{min}{ }^{-1}$. A step gradient of sodium sulfate up to $0.5 \mathrm{M}$ was applied and all fractions were tested for SlcC, ComC and SuyAB.

Active fractions of SlcC or ComC were salted up to $1.7 \mathrm{M}$ ammonium sulfate, the precipitate was spun off and the supernatant was subjected to hydrophobic interaction chromatography on Phenyl Superose HR 10/10 (Pharmacia). A linear decreasing gradient of ammonium sulfate in Tris/sulfate buffer, $\mathrm{pH} 8.5$, was applied. Samples were centrifuged in Vivaspin concentrators (10 kDa cut-off, Sartorius). Gel filtration on Superose 12 (HR 10/30, Pharmacia) was done in $50 \mathrm{mM}$ Tris/ sulfate buffer, $\mathrm{pH} 9.0$, including $150 \mathrm{mM}$ sodium sulfate, at a flow rate of $0.4 \mathrm{ml} \mathrm{min}{ }^{-1}$. Standard high molecular mass proteins (aprotinin, RNase A, carbonic anhydrase, ovalbumin, conalbumin, aldolase and ferritin) served to calibrate the column.

Analytical methods. Protein in whole cells was quantified by a Lowry-type method (Kennedy \& Fewson, 1968), omitting the initial acid treatment to avoid precipitation of salts originating from the medium. Soluble protein was assayed by protein-dye binding (Bradford, 1976). Denatured proteins were separated by $16 \%$ SDSPAGE gels and stained with Coomassie brillant blue R250 (Laemmli, 1970). Stained protein bands were cut out of the gel and subjected to peptide-mass fingerprinting to identify the corresponding genes (done under contract at TopLab, Martinsried, Germany). Sulfolactate and sulfopyruvate were quantified by ion chromatography with suppression (Denger et al., 2004). Sulfate release during growth was measured as the turbidity of a suspension of insoluble $\mathrm{BaSO}_{4}$ (Sörbo, 1987) with at least a 1:5 dilution to avoid precipitation with salts of the medium. Sulfite was quantified as the fuchsin adduct (Denger et al., 2001).

Various unsuccessful attempts were made to separate the enantiomers of sulfolactate by HPLC on a chiral column (Nucleosil Chiral-1 phase; Macherey-Nagel) according to the manufacturer's suggestions. Separations of the enantiomers of $(R, S)$-alanine and of $(R, S)$-lactate were achieved.

Software for sequence analysis. Analyses of the finished genome sequence (accession no. NC_007963, GenBank: CP000285) of C. salexigens DSM 3043 were done using the BLAST algorithm on the National Center for Biotechnology Information website (http://www. ncbi.nlm.nih.gov/) and the IMG page of JGI (http://img.jgi.doe.gov/ cgi-bin/pub/main.cgi). The BLAST server on the Transport Classification Database (http://www.tcdb.org/), which is coupled to a predictor of transmembrane helices, was also used. Sequence data were manipulated with different subroutines from the LASERGENE program package (DNASTAR).

\section{RESULTS}

\section{Growth of $C$. salexigens with $(R, S)$-sulfolactate}

C. salexigens DSM 3043 utilized $(R, S)$-sulfolactate as sole source(s) of carbon and energy for growth. Sulfolactate was utilized quantitatively with stoichiometric release of the sulfonate moiety as sulfate; about $25 \%$ of this sulfonate was released transiently as sulfite (Fig. 2 a, b). A molar growth yield of about $5 \mathrm{~g}$ protein (mol sulfolactate carbon) $)^{-1}$ was observed, typical for the quantitative utilization of carbon (Cook, 1987); apparently both enantiomers of sulfolactate were completely oxidized. The growth rate was $0.12 \mathrm{~h}^{-1}$ and a specific degradation rate for sulfolactate of $2.2 \mathrm{mkat}$ ( $\mathrm{kg}$ protein $)^{-1}$ could be calculated.

\section{Activities of the hypothesized enzymes in the dissimilation of sulfolactate}

The postulated pathway (Fig. 1) involved a threecomponent transport system for the polar sulfolactate, $(S)$-sulfolactate oxidoreductase (SlcC), (R)-sulfolactate oxidoreductase (ComC), desulfonative sulfolactate sulfolyase (SuyAB), an export system for sulfite and a putative sulfite dehydrogenase. The carbon skeleton would enter standard intermediary metabolism as pyruvate.

With crude extracts of sulfolactate-grown cells of $C$. salexigens, a specific enzyme activity of $(S)$-sulfolactate oxidoreductase (SlcC) of about 7.0 mkat $(\mathrm{kg} \text { protein })^{-1}$ was observed. With acetate-grown cells the activity was about 0.2 mkat $(\mathrm{kg} \text { protein })^{-1}$, which showed that the enzyme was inducible.

The specific activity of (R)-sulfolactate oxidoreductase (ComC) with crude extract of sulfolactate-grown cells was 0.8 mkat (kg protein $)^{-1}$. No activity was measurable with 


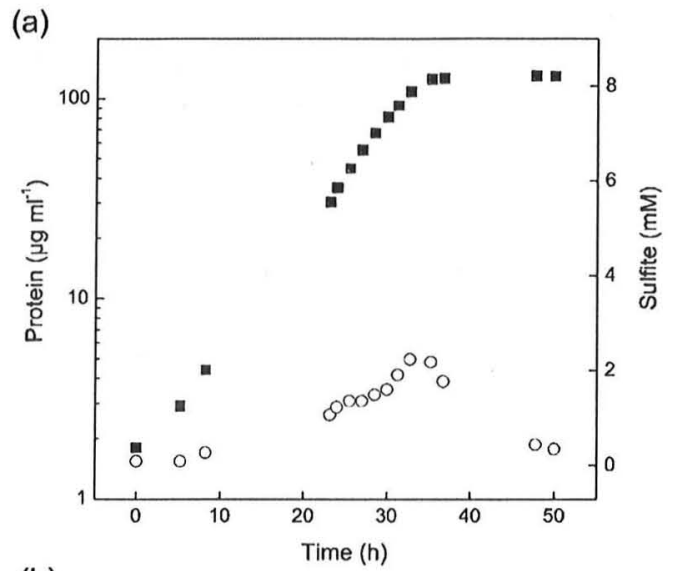

(b)

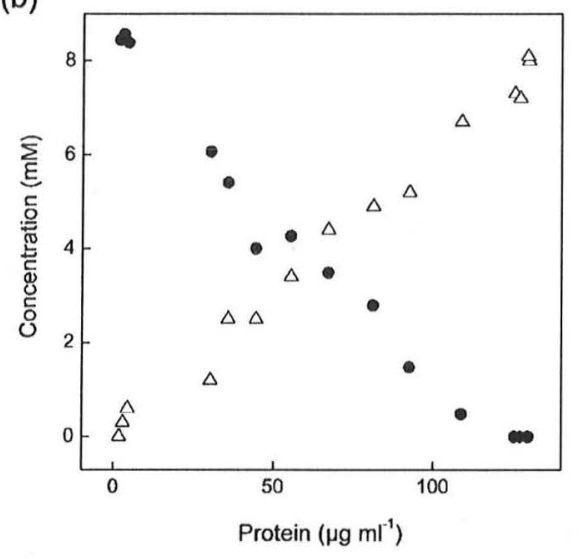

Fig. 2. Growth of $C$. salexigens with $(R, S)$-sulfolactate as carbon source, showing transient accumulation of sulfite (a), and the behaviour of substrate(s) and product as a function of growth (b). $\square$, Protein; $\bigcirc$, sulfite; $\bullet$, sulfolactate; $\triangle$, sulfate.

extracts of acetate-grown cells, suggesting inducibility of ComC.

Sulfolactate sulfo-lyase (SuyAB) could be detected in freshly prepared crude extract of sulfolactate-grown cells. The specific activity was 2.2 mkat $(\mathrm{kg} \text { protein })^{-1}$ in the crude extract and was found to be 3.3 mkat (kg protein $)^{-1}$ in the soluble fraction. No activity was detected in extracts of acetate-grown cells, so the enzyme activity was inducible. This enzyme was very unstable and was lost on freezing. Storage at $4{ }^{\circ} \mathrm{C}$ for 1 day resulted in a $50 \%$ loss of activity.

Sulfite dehydrogenase (SorAB) could not be measured in crude extracts with either ferricyanide or eukaryotic cytochrome $c$ as electron acceptor. This corresponded with the absence of appropriate gene candidates (sorAB) (Denger et al., 2008).

\section{Enrichment, purification or separation and identification of the relevant enzymes}

Proteins in crude extracts of acetate-grown cells or of sulfolactate-grown cells were separated by SDS-PAGE. Two strongly induced protein bands were observed (Fig. 3, lanes 1 and 2). The strong $43 \mathrm{kDa}$ band (SuyB) was anticipated (Fig. 1) (Rein et al., 2005) whereas the $30 \mathrm{kDa}$ band was a novelty.

Proteins in the soluble extract of sulfolactate-grown cells were loaded on to an anion-exchange column. ComC and SlcC eluted in that order in slightly overlapping fractions at about $120 \mathrm{mM}$ sulfate, and the $30 \mathrm{kDa}$ band could be observed in the SlcC fraction (Fig. 3, lane 3). A broad activity peak of SuyAB with a very low specific activity eluted at about $170 \mathrm{mM}$ sulfate.

The fraction with the highest activity of SlcC was subjected to hydrophobic interaction chromatography and the activity eluted after the end of the decreasing gradient. Gel filtration of SlcC followed as a further purification step, and to provide an estimate of the native molecular mass of the protein. SlcC was, thus, purified 91-fold to homogeneity with a recovery of $14 \%$ (Table 1; Fig. 3, lane 5). The denatured enzyme had a molecular mass of about $34 \mathrm{kDa}$ (Fig. 3). Native SlcC eluted from the gel filtration column with a retention time which indicated a molecular mass of about $61 \mathrm{kDa}$, suggesting a dimeric structure.

The MonoQ fraction with highest activity of ComC was also subjected to hydrophobic interaction chromatography. The enzyme eluted later than SlcC, and a 120 -fold enrichment was attained. The corresponding protein band on SDSPAGE indicated a molecular mass of $37 \mathrm{kDa}$ (not shown).

Samples from the anion-exchanger with SuyAB activity were separated by SDS-PAGE. The prominent band at 43 kDa (SuyB; see Fig. 3) was enriched (not shown).

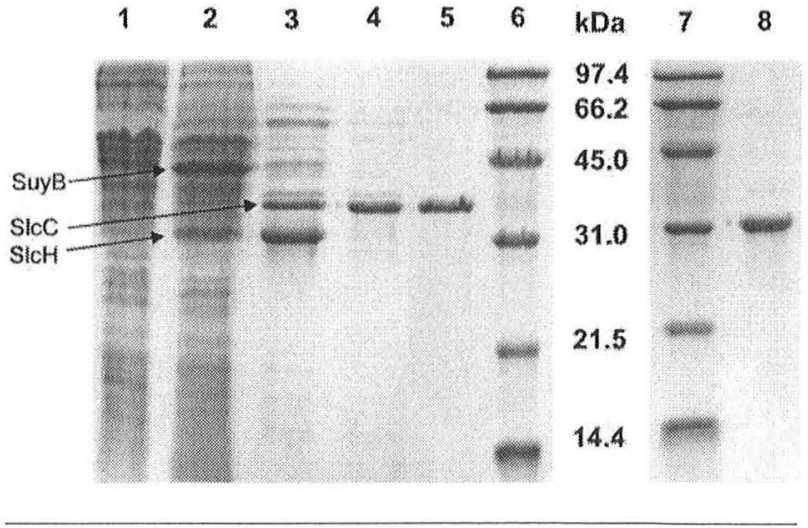

Fig. 3. Composite electropherograms (SDS-PAGE) of denatured proteins from different stages of the purification of SlcC from C. salexigens, with purified $\mathrm{SlcH}$. Lane 1, crude extract of acetategrown cells; lane 2, crude extract of sulfolactate-grown cells; lane 3 , active fraction after anion-exchange chromatography; lane 4, active fraction after hydrophobic interaction chromatography; lane 5 , purified enzyme (from a different preparation) after gel filtration chromatography; lanes 6 and 7, molecular mass markers (with masses in $\mathrm{kDa}$ ); lane 8, the purified, unknown, induced protein later identified as $\mathrm{SlcH}$. 
Table 1. Purification of SlcC from C. salexigens

\begin{tabular}{|lccccc|}
\hline Purification step & Total protein $(\mathbf{m g})$ & $\begin{array}{c}\text { Total activity } \\
\text { (nkat) }\end{array}$ & Recovery (\%) & $\begin{array}{c}\text { Specific activity [mkat } \\
\text { (kg protein) }^{-1} \text { ] }\end{array}$ & Purification (-fold) \\
\hline Crude extract & 147 & 1088 & 100 & 7.4 & 1 \\
Soluble fraction & 104 & 884 & 81 & 8.5 & 1 \\
Anion exchange & 4.2 & 722 & 66 & 172 & 23 \\
HIC* & 0.9 & 266 & 24 & 310 & 42 \\
Gel filtration & 0.2 & 157 & 14 & 675 & 91 \\
\hline
\end{tabular}

${ }^{*}$ HIC, hydrophobic interaction chromatography.

The unknown, inducible $30 \mathrm{kDa}$ protein co-eluted from the anion-exchanger with SlcC (Fig. 3, lanes 2 and 3). The $30 \mathrm{kDa}$ protein was essentially homogeneous in the following fraction (Fig. 3, lane 8).

The four separated proteins were excised from the SDS gels and subjected to peptide-mass fingerprinting. The data confirmed that the three enzymes did, indeed, represent the gene products of the candidate slcC gene (Csal_1770) (Fig. 1), the candidate com $C$ gene (Csal_1771) and the candidate suyB gene (Csal_1766). The unknown protein was identified as Csal_1767, the candidate periplasmic binding protein $(\mathrm{SlcH})$ of the sulfolactate transporter. We then compared separated (SDS-PAGE) membrane fractions of induced and non-induced cells, but there was no evidence for overexpressed membrane components (SlcFG) (not shown).

\section{Some properties of the purified dehydrogenases}

Both SlcC and ComC were stable at $4{ }^{\circ} \mathrm{C}$ for several weeks. Each could be frozen and thawed without loss of activity.

Purified SlcC converted sulfopyruvate into sulfolactate as demonstrated by ion chromatography; substrate and product were stable under the reaction conditions as shown by the controls without enzyme (Fig. 4). SlcC accepted NADH as co-substrate in this reaction. The $K_{\mathrm{m}}^{\mathrm{app}}$ value for $\mathrm{NADH}$ was estimated as $<50 \mu \mathrm{M}$, that for sulfopyruvate as $<140 \mu \mathrm{M}$. Neither pyruvate nor oxaloacetate $(5 \mathrm{mM})$ was a substrate, and neither compound was inhibitory.

SlcC could also be assayed in the forward direction, by trapping sulfopyruvate with hydrazine. Only $\mathrm{NAD}^{+}$(and not $\mathrm{NADP}^{+}$) was a co-substrate. $K_{\mathrm{m}}^{\text {app }}$ values for $\mathrm{NAD}^{+}$ and $(R, S)$-sulfolactate were estimated as $2 \mathrm{mM}$ and $7 \mathrm{mM}$, respectively. The specific activity under these conditions was only about $5 \%$ of that in the reverse direction. The automatic annotation of $s l c C$ was ' $(S)$-3-phosphoglycerate dehydrogenase'. We tested $(S)$-3-phosphoglycerate, $(R)$ malate, $(R)$-lactate, $(S)$-malate and $(S)$-lactate and none was a substrate for SlcC (and none was inhibitory). We compared the sequences of 14 candidate SlcCs (see below) with those of characterized 3-phosphoglycerate dehydrogenases [EC 1.1.1.95] in a dendrogram, and found that they clustered separately (not shown). SlcC is an enzyme in its own right.

ComC also converted sulfopyruvate into sulfolactate (data not shown). Neither pyruvate nor oxaloacetate (5 mM) was a substrate. NADPH served as co-substrate. The $K_{\mathrm{m}}^{\mathrm{app}}$ value for NADPH was about $100 \mu \mathrm{M}$, that for sulfopyruvate about $140 \mu \mathrm{M}$. In the assay in the oxidative direction with hydrazine buffer, $\mathrm{NADP}^{+}$could not be replaced by $\mathrm{NAD}^{+}$. Neither $(S)$-malate, $(S)$-lactate, $(R)$-malate nor $(R)$-lactate was a substrate for ComC (and none inhibited the enzyme). $K_{\mathrm{m}}^{\mathrm{app}}$ values of $1.2 \pm 0.02 \mathrm{mM}$ for $\mathrm{NADP}^{+}$ and $19 \pm 5 \mathrm{mM}$ for sulfolactate were calculated.

\section{Coupling SIcC or ComC to SuyAB}

We were able to separate the enantiomers of alanine and lactate on a commercially available HPLC column, but we found no conditions which separated the enantiomers of $(R, S)$-sulfolactate. So we deduced the stereochemistry involved from the thorough work done elsewhere with ComC (Graham \& White, 2002; Graupner et al., 2000).

Sulfopyruvate, NADH and SlcC were incubated for $15 \mathrm{~min}$, after which separated SuyAB was added. There

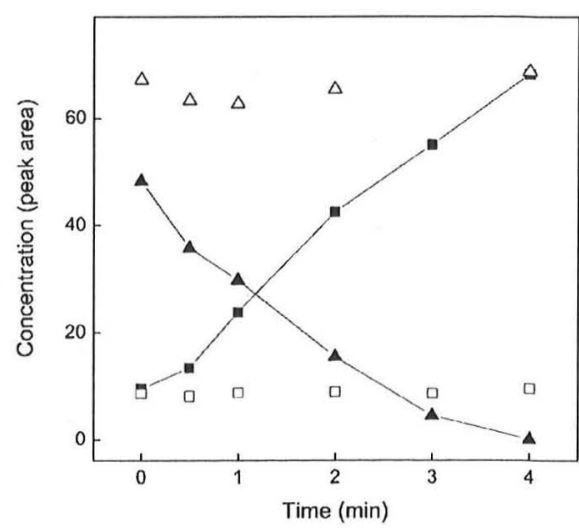

Fig. 4. Conversion of $0.5 \mathrm{mM}$ sulfopyruvate $(\mathbf{\Lambda})$ to sulfolactate ( $\square$ ) by SlcC. In control experiments, sulfopyruvate $(\Delta)$ or sulfolactate ( $\square$ ) was stable in the absence of enzyme. 
was negligible formation of sulfite. In contrast, after preincubation of sulfopyruvate, NADPH and ComC, addition of SuyAB led to release of significant amounts of sulfite. ComC generates $(R)$-sulfolactate from sulfopyruvate (Graupner et al., 2000), so we deduce that (R)sulfolactate is the substrate for SuyAB. Correspondingly, the sulfolactate, which is generated by $\mathrm{SlcC}$ from sulfopyruvate (Fig. 4) and which is not a substrate for SuyAB, must be $(S)$-sulfolactate.

\section{Bioinformatic data}

There are currently 13 genomes with a suyAB-slcC-comC cluster (Table 2), and several more with $\operatorname{slcC}$ or $\operatorname{comC}$ elsewhere on the chromosome. Representative organisms from Table 2 were tested for growth with sulfolactate. Methylobacterium radiotolerans and Oligotropha carboxidovorans grew with the compound and excreted sulfate, whereas Cupriavidus necator and Rhodococcus jostii did not grow.

\section{DISCUSSION}

Growth of $C$. salexigens with sulfolactate, detected elsewhere (Denger et al., 2009), is seen to be quantitative (Fig. 2a, b). The release of sulfite into the medium (Fig. 2a) indicates the presence of an unidentified sulfite exporter in the cytoplasmic membrane. The unidentified sulfite dehydrogenase, which we place in the periplasm (Denger et al., 2008; Kappler et al., 2000), completes the pathway (Fig. 1).
The pathway is inducible (e.g. Fig. 3). No regulatory work has been done, but the putative GntR-type transcriptional regulator, SuyR, first discovered contiguous with $s u y A B$ in Paracoccus denitrificans NKNCYSA (Rein et al., 2005), is also found in all organisms in Table 2. So the hypothesis for the role of SuyR is still valid.

The initial step in the pathway is an uptake system, SlcHFG [TC 2.A.80.---] (Fig. 1). When first proposed in $R$. nubinhibens (Denger et al., 2009), the experimental support for SlcHFG was from reverse-transcription PCR. We now have strong expression of one component, SlcH (Fig. 3), as proof that at least one of the genes is transcribed. However, we still do not know exactly what is transported. Is only one enantiomer of $(R, S)$-sulfolactate transported, or both? If the former, there must be a second transporter, whose identity is unknown.

SlcHFG is a rare transporter (Denger et al., 2009), being found in organisms in Table 2 only in C. salexigens, Fulvimarina pelagi (some five to seven genes upstream) and possibly in Oligotropha carboxidovorans. Alternative uptake mechanisms seem to be DAACS and ABC transporters (Table 2). Many organisms seem to have no transporter for sulfolactate (Table 2), and sulfolactate does not support growth (see Results). In the latter cases, we presume that external sulfolactate is not utilized (e.g. Rhodococcus jostii RHA1), because a transport system is not available, and that any sulfolactate in the cell is generated intracellularly from a precursor, analogous to the situation in Ruegeria pomeroyi DSS-3, where the sulfolactate is apparently

Table 2. Organisms containing the suyAB and s/cC-comC cluster

Only C. salexigens has the slcHFG genes in this cluster; F. pelagi has the genes for this transporter elsewhere on the genome, and most organisms encode no transporter at this position. All organisms encode a putative transcriptional regulator (SuyR) at one or other end of the cluster. The acronyms 'DAACS' and 'ABC transporter' represent the dicarboxylate/amino acid: cation symporter family [TC 2.A.23.-.-] and the ATP binding cassette superfamily [TC 3.A.1.---].

\begin{tabular}{|c|c|c|c|c|c|c|c|}
\hline Organism & Tag & suyR & suyA & suyB & $\begin{array}{l}\text { Genes between } \\
\text { suyB and slcC }\end{array}$ & slcC & $\operatorname{com} C$ \\
\hline Chromohalobacter salexigens DSM 3043 & Csal__ & 1764 & 1765 & 1766 & slcHFG & 1770 & 1771 \\
\hline Cupriavidus necator JMP134 & Reut_ & B3533 & B3528 & B3529 & None & B3530 & B3531 \\
\hline Cupriavidus taiwanensis LMG 19424 & RALTA_ & B0844 & B0839 & B0840 & None & B0841 & B0842 \\
\hline Fulvimarina pelagi HTCC 2506 & FP2506_1 & 14334 & 14329 & 14324 & None & 14319 & 14314 \\
\hline Hoeflea phototrophica DFL42 & HPDFL43_ & 17106 & 17101 & 17096 & None & 17091 & 17086 \\
\hline Herminiimonas arsenicoxydans & HEAR & 3334 & 3336 & 3337 & $\begin{array}{c}\text { Encode an } \mathrm{ABC} \\
\text { transporter? }\end{array}$ & 3340 & 3341 \\
\hline Meiothermus ruber & MrubDRAFT_ & 06820 & 06830 & 06840 & None & 06850 & 06860 \\
\hline Methylobacterium radiotolerans DSM 1819 & Mrad2831_ & 5975 & 5980 & 5979 & $\begin{array}{l}\text { Encode a DAACS } \\
\text { transporter }\end{array}$ & 5977 & 5976 \\
\hline Oligotropha carboxidovorans OM5 & OCAR_ & 7559 & 7558 & 7557 & Variant slcHFG & 7552 & 7551 \\
\hline Rhodococcus jostii RHA1 & RHA1_ro & 08849 & 08848 & 08847 & No transport genes & 08844 & 08843 \\
\hline Variovorax paradoxus $\mathrm{S} 110$ & Vapar_ & 1590 & 3818 & 3819 & None & 3820 & 3821 \\
\hline Verminephrobacter eiseniae & Veis_ & 0894 & 0893 & 0892 & None & 0891 & 0890 \\
\hline Bordetella petrii & Bpet & 0416 & 0415 & 0414 & $\begin{array}{c}\text { Encode an } \mathrm{ABC} \\
\text { transporter }\end{array}$ & 0409 & 0408 \\
\hline
\end{tabular}


generated from 2,3-dihydroxypropanesulfonate (Denger et al., 2009).

In contrast to the incomplete story (stories) at the cell surface, there is clarity about the reactions of the scalar enzymes in the pathway. The novelty is the $(S)$-sulfolactate dehydrogenase (SlcC) (Fig. 1). Its function is to convert the $(S)$-enantiomer into sulfopyruvate, which allows ComC to convert sulfopyruvate into $(R)$-sulfolactate, the substrate for the desulfonation reaction, SuyAB. The novel enzyme belongs to [EC 1.1.1.-] in the Enzyme List, and we suggest the systematic name ' $(S)$-sulfolactate: $\mathrm{NAD}^{+}$oxidoreductase'. The accepted name could be $(S)$-sulfolactate dehydrogenase.

There are, thus, at least three sulfolactate oxidoreductases. The best-characterized is ComC [EC 1.1.1.272] in the biosynthesis of coenzyme $\mathrm{M}$ in Methanococcus jannaschii (and methanogens in general) (Graham \& White, 2002; Graupner et al., 2000) and in spore-formers. The leastunderstood is SlcD [EC 1.1.99.-], a membrane-bound enzyme in the degradation of sulfolactate in R. nubinhibens ISM (Denger et al., 2009). Finally, there is SlcC, with its unusual role as a partial racemase in a degradative pathway, which is introduced here.

The observation of two dehydrogenases effecting racemization seems to be novel. Others have used the hypothesis that this could occur, but their experimental data showed that a different metabolic route was used (Haupt \& Bocker, 1965; Lincoln et al., 1987).

There are about 50 orthologues of $\operatorname{suy} A B$ in the open databases. Only about $30 \%$ of these orthologues (Table 2) are in organisms which seem to involve racemization via $\mathrm{SlcC}$ and ComC as in Fig. 1. Other orthologues (30\%) are found in thermophilic archaea, all of which have a potential racemase encoded adjacent to $s u y B$. The other orthologues of suyAB are found in several different clusters (not shown). The diversity in pathways of sulfonate degradation (e.g. Baldock et al., 2007; Denger et al., 2009; Weinitschke et al., 2010) is, thus, recurrent, and indicates how widespread the sulfonates must be in marine and terrestrial environments.

\section{ACKNOWLEDGEMENTS}

We are grateful to Gregor Habeck for data generated in an advanced practical class. Funding was provided by the University of Konstanz. We are grateful to many sequencing organizations, but especially to the DOE Joint Genome Institute for sequencing the genome of $C$. salexigens DSM 3043 and for making the data generally available.

\section{REFERENCES}

Arahal, D. R., Garcia, M. T., Vargas, C., Canovas, D., Nieto, J. J. \& Ventosa, A. (2001). Chromohalobacter salexigens sp. nov., a moderately halophilic species that includes Halomonas elongata DSM 3043 and ATCC 33174. Int J Syst Evol Microbiol 51, 1457-1462.
Baldock, M. I., Denger, K., Smits, T. H. M. \& Cook, A. M. (2007). Roseovarius sp. strain 217: aerobic taurine dissimilation via acetate kinase and acetate-CoA ligase. FEMS Microbiol Lett 271, 202-206.

Benning, C. (2007). Questions remaining in sulfolipid biosynthesis: a historical perspective. Photosynth Res 92, 199-203.

Benson, A. S. \& Lee, R. F. (1972). The sulphoglycolytic pathway in plants. Biochem J 128, 29P-30P.

Bonsen, P. P. M., Spudich, J. A., Nelson, D. L. \& Kornberg, A. (1969). Biochemical studies of bacterial sporulation and germination. XII. A sulfonic acid as a major sulfur compound of Bacillus subtilis spores. $J$ Bacteriol 98, 62-68.

Bradford, M. M. (1976). A rapid and sensitive method for the quantitation of microgram quantities of protein utilizing the principle of protein-dye binding. Anal Biochem 72, 248-254.

Cook, A. M. (1987). Biodegradation of s-triazine xenobiotics. FEMS Microbiol Rev 46, 93-116.

Danko, A. S., Saski, C. A., Tomkins, J. P. \& Freedman, D. L. (2006). Involvement of coenzyme $M$ during aerobic biodegradation of vinyl chloride and ethene by Pseudomonas putida strain AJ and Ochrobactrum sp. strain TD. Appl Environ Microbiol 72, 3756-3758.

Denger, K., Ruff, J., Rein, U. \& Cook, A. M. (2001). Sulfoacetaldehyde sulfo-lyase (EC 4.4.1.12) from Desulfonispora thiosulfatigenes: purification, properties and primary sequence. Biochem J 357, 581-586.

Denger, K., Weinitschke, S., Hollemeyer, K. \& Cook, A. M. (2004). Sulfoacetate generated by Rhodopseudomonas palustris from taurine. Arch Microbiol 182, 254-258.

Denger, K., Smits, T. H. M. \& Cook, A. M. (2006). L-Cysteate sulpholyase, a widespread, pyridoxal $5^{\prime}$-phosphate-coupled desulphonative enzyme purified from Silicibacter pomeroyi DSS $-3^{\mathrm{T}}$. Biochem $J 394$, 657-664.

Denger, K., Weinitschke, S., Smits, T. H. M., Schleheck, D. \& Cook, A. M. (2008). Bacterial sulfite dehydrogenases in organotrophic metabolism: separation and identification in Cupriavidus necator $\mathrm{H} 16$ and in Delftia acidovorans SPH-1. Microbiology 154, 256-263.

Denger, K., Mayer, J., Buhmann, M., Weinitschke, S., Smits, T. H. M. \& Cook, A. M. (2009). Bifurcated degradative pathway of 3-sulfolactate in Roseovarius nubinhibens ISM via sulfoacetaldehyde acetyltransferase and (S)-cysteate sulfo-lyase. J Bacteriol 191, 5648-5656.

Folkers, K., Koniuszy, F. \& Shavel, J. (1944). Erythrina alkaloids. XIV. Isolation and characterization of erysothiovine and erysothiopine, new alkaloids containing sulfur. J Am Chem Soc 66, 1083-1087.

Graham, D. E. \& White, R. H. (2002). Elucidation of methanogenic coenzyme biosyntheses: from spectroscopy to genomics. Nat Prod Rep 19, 133-147.

Graupner, M., Xu, H. \& White, R. H. (2000). Identification of an archaeal 2-hydroxy acid dehydrogenase catalyzing reactions involved in coenzyme biosynthesis in methanoarchaea. J Bacteriol 182, 36883692 .

Haupt, I. \& Bocker, H. (1965). On formation of DL-alanine in cultures of a mutant of Streptomyces albus var. metamycinus JA 3626. Hoppe Seylers Z Physiol Chem 342, 132-139.

Irimia, A., Madern, D., Zaccai, G. \& Vellieux, F. M. D. (2004). Methanoarchaeal sulfolactate dehydrogenase: prototype of a new family of NADH-dependent enzymes. EMBO J 23, 1234-1244.

Junker, F., Field, J. A., Bangerter, F., Ramsteiner, K., Kohler, H.-P., Joannou, C. L., Mason, J. R., Leisinger, T. \& Cook, A. M. (1994). Oxygenation and spontaneous deamination of 2-aminobenzenesulphonic acid in Alcaligenes sp. strain O-1 with subsequent meta ring cleavage and spontaneous desulphonation to 2-hydroxymuconic acid. Biochem J 300, 429-436. 
Kappler, U., Bennett, B., Rethmeier, J., Schwarz, G., Deutzmann, R., McEwan, A. G. \& Dahl, C. (2000). Sulfite: cytochrome $c$ oxidoreductase from Thiobacillus novellus. Purification, characterization, and molecular biology of a heterodimeric member of the sulfite oxidase family. J Biol Chem 275, 13202-13212.

Kennedy, S. I. T. \& Fewson, C. A. (1968). Enzymes of the mandelate pathway in Bacterium N.C.I.B. 8250. Biochem J 107, 497-506.

Krejčik, Z., Denger, K., Weinitschke, S., Hollemeyer, K., Pačes, V., Cook, A. M. \& Smits, T. H. M. (2008). Sulfoacetate released during the assimilation of taurine-nitrogen by Neptuniibacter caesariensis: purification of sulfoacetaldehyde dehydrogenase. Arch Microbiol 190, 159-168.

Laemmli, U. K. (1970). Cleavage of structural proteins during the assembly of the head of bacteriophage T4. Nature 227, 680-685.

Lee, R. F. \& Benson, A. A. (1972). The metabolism of glyceryl $\left[{ }^{35} \mathrm{~S}\right]$ sulfoquinovoside by the coral tree, Erythrina crista-galli, and alfalfa, Medicago sativa. Biochim Biophys Acta 261, 35-37.

Lincoln, B. C., Des Rosiers, C. \& Brunengraber, H. (1987). Metabolism of S-3-hydroxybutyrate in the perfused rat liver. Arch Biochem Biophys 259, 149-156.

Metzler, D. E. (2003). Biochemistry: The Chemical Reactions of Living Cells, 2nd edn. Amsterdam: Academic Press.

Pfennig, N. (1978). Rhodocyclus purpureus gen. nov. sp. nov., a ring-shaped, vitamin $B_{12}$-requiring member of the family Rhodospirillaceae. Int J Syst Bacteriol 28, 283-288.

Reichenbecher, W., Kelly, D. P. \& Murrell, J. C. (1999). Desulfonation of propanesulfonic acid by Comamonas acidovorans strain P53: evidence for an alkanesulfonate sulfonatase and an atypical sulfite dehydrogenase. Arch Microbiol 172, 387-392.
Rein, U., Gueta, R., Denger, K., Ruff, J., Hollemeyer, K. \& Cook, A. M. (2005). Dissimilation of cysteate via 3 -sulfolactate sulfo-lyase and a sulfate exporter in Paracoccus pantotrophus NKNCYSA. Microbiology 151, 737-747.

Roy, A. B., Hewlins, M. J. E., Ellis, A. J., Harwood, J. L. \& White, G. F. (2003). Glycolytic breakdown of sulfoquinovose in bacteria: a missing link in the sulfur cycle. Appl Environ Microbiol 69, 6434-6441.

Ruff, J., Denger, K. \& Cook, A. M. (2003). Sulphoacetaldehyde acetyltransferase yields acetyl phosphate: purification from Alcaligenes defragrans and gene clusters in taurine degradation. Biochem J 369, 275-285.

Shibuya, I., Yagi, T. \& Benson, A. A. (1963). Sulfonic acids in algae. In Studies on Microalgae and Photosynthetic Bacteria, pp. 627-636. Edited by Japanese Society of Plant Physiologists. Tokyo: The University of Tokyo Press.

Sörbo, B. (1987). Sulfate: turbidimetric and nephelometric methods. Methods Enzymol 143, 3-6.

Thurnheer, T., Köhler, T., Cook, A. M. \& Leisinger, T. (1986). Orthanilic acid and analogues as carbon sources for bacteria: growth physiology and enzymic desulphonation. J Gen Microbiol 132, 12151220

Weinitschke, S., Sharma, P. I., Stingl, U., Cook, A. M. \& Smits, T. H. M. (2010). Gene clusters involved in isethionate degradation in terrestrial and marine bacteria. Appl Environ Microbiol 76, 618-621.

Weinstein, C. L. \& Griffith, O. W. (1988). Cysteinesulfonate and $\beta$ sulfopyruvate metabolism. Partitioning between decarboxylation, transamination, and reduction pathways. J Biol Chem 263, 37353743.

Edited by: M. A. Kertesz 